\title{
Biochemical and Molecular Analysis of a Transmembrane Proteln KInase from Arabidopsis thaliana
}

\author{
Progress Report \\ for Grant No. DE-FG02-91ER20029
}

DOE/ER/20029--Tl

DE92 015078

\author{
A. B. Bleecker
}

\section{Introduction:}

Receptor protein kinases on the plasma membrane of cells interact with various growth factors and hormonal agents, generating intracellular signals from these external signals. As such the receptors play a pivotal role in the ability of a cell to respond to its environment and develop. Although widely studied in animals, very little is known about receptor kinases in plants.

We have isolated genomic and CDNA clones encoding a novel receptor-like protein kinase (LC5) from the higher plant Arabidopsis thaliana. The product of the LC5 gene has all the features expected in a receptor protein kinase: an amino-terminal signal sequence for targeting to a membrane, an extracellular receptor domain, a hydrophobic transmembrane domain, and an intracellular kinase domain. Two features of LC5 are of particular interest. First, the extracellular domain has 11 copies of a leucine-rich repeat (LRiR) sequence. Leucine rich repeats have only been found in proteins associated with the plasma membrane, and are implicated in protein/protein interactions. No protein with leucine rich repeats has previously been identified or characterized in plants. Second, the kinase domain of LC5 appears to code for a serine/threonine specific kinase, not a tyrosine specific kinase as is typical of the animal receptor kinases. To date, only three other transmembrane protein kinases have been identified with serine/threonine specific sequences and none have been characterized biochemically. We are following biochemical, molecular, and genetic approaches in characterizing the LC5 kinase and the role that it plays in plant signal transduction.

\section{Progress Summary:}

Immunodetection of LC5 protein in Arabidopsis. The LRR domain and a portion of the kinase domain were individually cloned into an expression vector PGEX2T, and recombinant fusion proteins were used to obtain domainspecific polyclonal antibodies. Total leaf extracts and the membrane fraction of Arabidopsis were probed with the domain-specific antibodies. A single immunodecorated band was detected with both antibodies at $M_{r}=120,000$. A variety of Arabidopsis tissue types were probed with the polyclonal antibody to

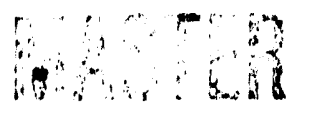
the LRR domain, and the LC5 polypeptide was detected in all tissue types exainined (root, stem, leaf, silique, and flower). The antibody is ncw being used 
for immunocytochemical localization, and preliminary results on stem sections indicate the presence of LC5 in all living cell types with highest levels of expression in outer cells of the cortex and in phloem cells. Lower levels of expression were observed in inner cells of the cortex and in the pith.

GUS reporter gene analysis of LC5. As a compliment to immunocytochemical localization, we are also attempting to obtain more explicit information as to what cell types LC5 is expressed in by use of the $\beta$ glucuronidase (GUS) reporter gene system. We have inserted the promoter region of the LC5 gene in front of the reporter gene GUS on a plasmid, and we are now in the process of transforming Arabidopsis with this construct. The expression of GUS in transformed plants can be determined histochemically by incubating plant tissue with X-GLUC, which results in a deep blue color appearing within the expressing cells.

Glycosylation of the LC5 polypeptide in Arabidopsis. All the known animal receptor kinases are glycosylated and, taking advantage of their carbohydrate moieties, can be partially purified on lectin columris. Arabidopsis membranes were solubilized with $1 \%$ Triton $X-100$ and incubated with various immobilized lectins. The immunodecorated LC5 polypeptide bound to concanavalin A-agarose like most plant glycoproteins which typically contain mannose in the oligosaccharide chain. We found that the LC5 polypeptide could also bind and be specifically eluted from wheat germ lectin-agarose which binds $\mathrm{N}$-acetylglucosamine residues. These results demonstrated that LC5 is a glycoprotein, and suggested that it might not contain solely the typical high-mannose glycans found in many plant glycoproteins, but might instead contain glycans of a more complex type.

We examined glycosylation of the LC5 polypeptide expressed in Arabidonsis in more detail. A $1 \%$ Triton X-100 extract of Arabidopsis was denatured, and then digested with endoglycosidase-F which will cleave at mannose residues in the glycan. Treatment with endoglycosidase-F reduced the apparent molecular mass of the immunodecorated polypeptide by 10,000 daltons, further demonstrating that LC5 is a glycoprotein. Unexpectedly, we also found that treatment with neuraminidase reduced the apparent molecular mass of the polypeptide by about 8,000 daltons. Neuraminidase removes sialic acid residues from oligosaccharide chains, however there have been no previous indications in the plant literature that plants have sialic acid substitution of their oligosaccharide chains.

Protein kinase activity in Arabidopsis. As mentioned previousiy, the animal receptor kinases are typically glycosylated and can be partially purified on lectin columns. We have examined the subpopulation of Arabidopsis proteins bound to the wheat germ lectin and the concanavalin A column for kinase activity. We find that kinase activity is present in this fraction of proteins and that several proteins are phosphorylated when a kinase assay is performed. Of particular interest is that many of these polypeptides are in the size range of 110,000 to 150,000 daltons, a size range typical for the receptor 
kinases. In addition, as with the animal receptor kinases, the phosphorylation of a number of these proteins showed greater stimulation by manganese than by magnesium.

We are also examining kinase activity of glycosylated membrane proteins in Arabidopsis after blotting to nitrocellulose membranes. In this procedure proteins are separated by SDS-polyacrylamide gel electrophoresis, blotted to nitrocellulose, and renatured on the blot, where the separated proteins can then be analyzed for kinase activity. We have used this procedure with the glycosylated membrane proteins purified by binding to the concanavalin $A$ column. We identify three polypeptides that renature with kinase activity and autophosphorylate. The most prominent of these is at the same molecular weight as the LC5 polypeptide.

Expression of LC5 kinase in E. coli. In addition to examining the activity of the native LC5 kinase in Arabidopsis, we are also attempting to overexpress the LC5 kinase in E. coli. We have inserted the kinase portion of LC5 into the vector pMAL-p, producing a fusion of the kinase with maltose-binding protein, and transformed this construct into $E$. coli. The fusion protein produced can then be rapidly purified away from other $E$. coli proteins by binding to an amylose affinity column. Upon induction of $E$. coli carrying our fusion construct, a polypeptide is produced of the expected molecular weight to be a fusion of the maltose-binding protein with the kinase domain of LC5. This polypeptide is also recognized by antibodies made against the kinase domain. Preliminary results indicate the the fusion protein produced has kinase activity and is capable of autophosphorylation. By use of this system we should be able to obtain large enough quantities of purified LC5 kinase to allow a careful biochemical analysis.

Transformation of LC5 into plants. As a means to better understanding the natural role of LC5 in the plant, we are performing plant transformations with various LC5 constructs. We currently have several independent transformations with the entire LC5 gene behind the soy heat shock promoter \#6871. By transforming with the entire LC5 gene we hope to see what effect overexpression of the protein has upon the plant. Other constructs which we are interested in transforming into plants include just the receptor region and just the kinase region of LC5.

\section{Incremental Request:}

In the coming year, we plan to pursue the research plans described in the original proposal. However, as a result of our recent findings, we also wish to propose an additional project. We now have evidence by following two lines of investigation that the LC5 polypeptide has kinase activity and is capable of autophosphorylation. We have been able to demonstrate this by purification of the native protein from Arabidopsis, where we have renatured the kinase with activity after blotting to nitrocellulose. In addition, we have overexpressed the 
kinase domain of LC5 as a fusion protein in E. coli. After affinity purification of the fusion protein, we also observe kinase activity and autophosphorylation. The ubservation that LC5 autophosphorylates is particularly interesting as autophosphorylation is an important means of regulation for many of the animal receptor kinases. Autophosphorylation has been found to directly affect kinase activity by modifying the enzyme kinetics (Ellis, L., Clauser, E., Morgan, D.O., Edery, M., Roth, R.A., and Rutter, W.J. (1986) Cell 45, 721-732; Yarden, Y., and Ullrich, A. (1988) Annu. Rev. Biochem. 57, 443-478), and has also been found to mediate binding between the kinase and specific intracellular proteins (Kazlauskas, A., and Cooper, J.A. (1989) Cell 58, 1121-1133).

In light of the importance of autophosphorylation to the receptor protein kinases, we propose to identify the autophosphorylation sites of the LC5 kinase. This should have significance not only to our understanding of this particular receptor kinase, but may have implications to other members of this receptor kinase family with serine/threonine specificity, none of which have been characterized biochemically. To determine the sites of phosphorylation we will make use of both the native renatured kinase and the fusion protein in $E$. coli. These purified proteins will be allowed to autophosphorylate in vitro using $\gamma 32 \mathrm{P}-\mathrm{ATP}$, and then digested with trypsin. Similar experiments using the animal receptor kinases have indicated that the same sites of autophosphorylation are used both in vivo and in vitro (Hsu, C.-Y.J., Mohammadi, M., Nathan, M., Honegger, A., Ullrich, A., Schlessinger, J., and Hurwitz, D.R. (1990) Cell Growth Differ. 1, 191-200); this could be confirmed for the LC5 kinase by phosphopeptide mapping after phosphorylation in vivo with $32 \mathrm{P}_{\mathrm{i}}$. The phosphorylated tryptic peptides will then be purified by reverse phase HPLC using a C-18 analytical column, and the amino acid sequence for each phosphorylated peptide determined by automated Edman degradation, the phosphorylated amino acid identified by the radiolabel. Identification of the sites of autophosphorylation will provide the basis for future research to evaluate the affect of autophosphorylation upon kinase activity and signal transduction.

The research associate (G. Eric Schaller) working on this project has previous experience with the isolation and sequencing of tryptic peptides (Schaller, G.E., and Sussman, M.R. (1988) Plant Physiol. 86, 512-516). In addition, there is an excellent on-campus Protein Sequence Facility at the University of Wisconsin Biotechnology Center which is capable of high sensitivity sequencing at the 1-10 pmole range. The typical cost for a single amino-acid sequence is $\$ 500.00$. To cover the costs of this additional proposed research, we wish to request an additional $\$ 3000$ beyond the planned budget for the next grant year of $\$ 77,000$. This would bring the total budge $l$ for the next grant year to $\$ 80,000$. 


\title{
Project Summary:
}

\section{Biochemical and Molecular Analysis of a Transmembrane Protein Kinase from Arabidopsis thaliana}

\author{
A. B. Bleecker, Department of Botany
}

Receptur protein kinases on the plasma membrane of cells interact with various growth factors and hormonal agents, generating intracellular signals from these external signals. As such the receptors play a pivotal role in the ability of a cell to respond to its environment and develop. Although widely studied in animals, very little is known about receptor kinases in plants.

We have isolated genomic and CDNA clones encoding a novel receptor-like protein kinase from the higher plant Arabidopsis thaliana. This kinase is being studied by combining biochemical, molecular, and genetic approaches. Domain-specific antibodies immunodecorate a polypeptide with a molecular mass of 120,000 daltons in extracts of Arabidopsis, where it has been found in all portions of the plant examined including root, stem, leaf, flower, and silique. Cytochemical analysis and studies using the kinase promoter with the GUS reporter gene system should give more explicit information on localization. The kinase is glycosylated, like the animal receptor kinases, and has been partially purified from Arabidopsis by using lectin columns. We have been able to demonstrate kinase activity by (1) renaturing the native protein from Arabidopsis after blotting to nitrocellulose, and (2) expressing the kinase in $E$. coli. Transgenic plants are also now being produced that either overexpress or carry altered forms of the protein kinase gene. These experiments will help determine the natural role the kinase plays in a pathway of signal transduction.

\section{DISCLAIMER}

\footnotetext{
This report vas prepared as an account of work sponsored by an agency of the United States Government. Neither the United States Government nor any agency thereof, nor any of their employees, makes any warranty, express or implied, or assumes any legal liability or responsibility for the accuracy, completeness, or usefulness of any information, apparatus, product, or process disclosed, or represents that its use would not infringe privately owned rights. Reference herein to any specific commercial preduct, process, or service by trade name, trademark, manufacturer, or otherwise does not necessarily constitute or imply its endorsement, recommendation, or favoring by the United States Government or any agency thereof. The views and opinions of authors expressed herein do not necessarily state or reflect those of the United States Government or any agency thereof.
} 


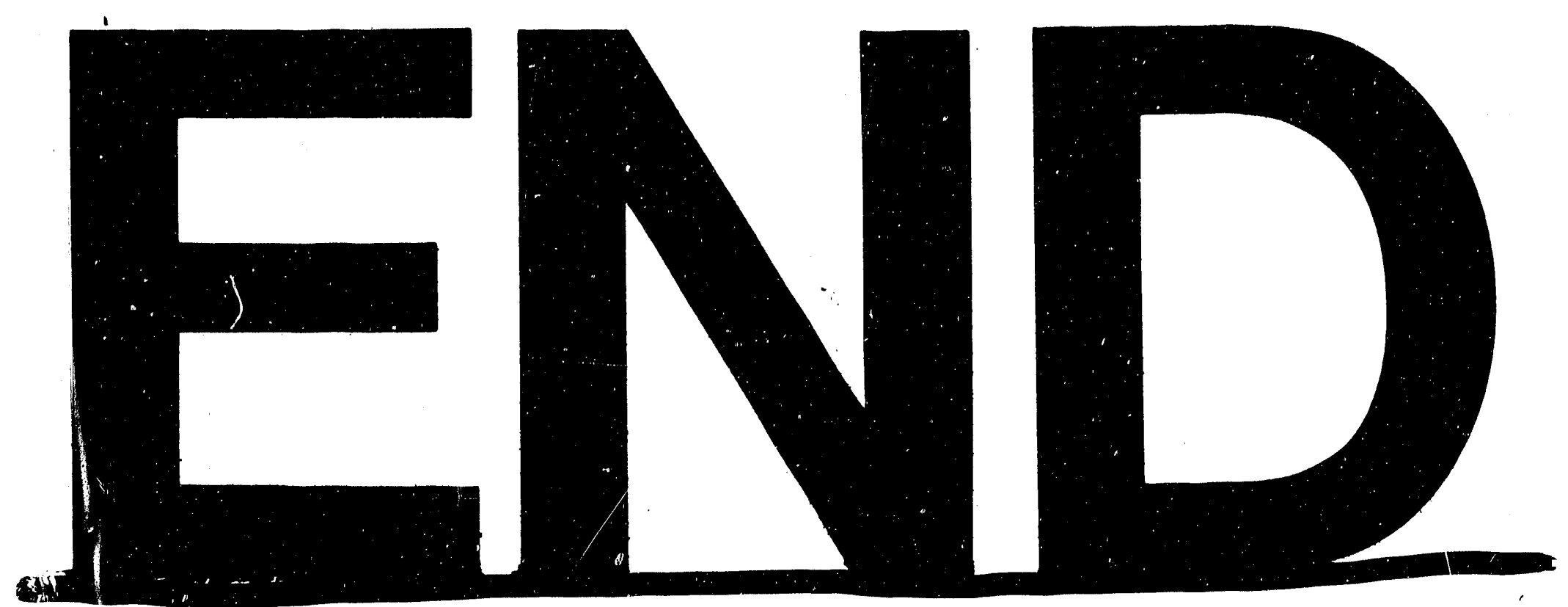

$\Delta$
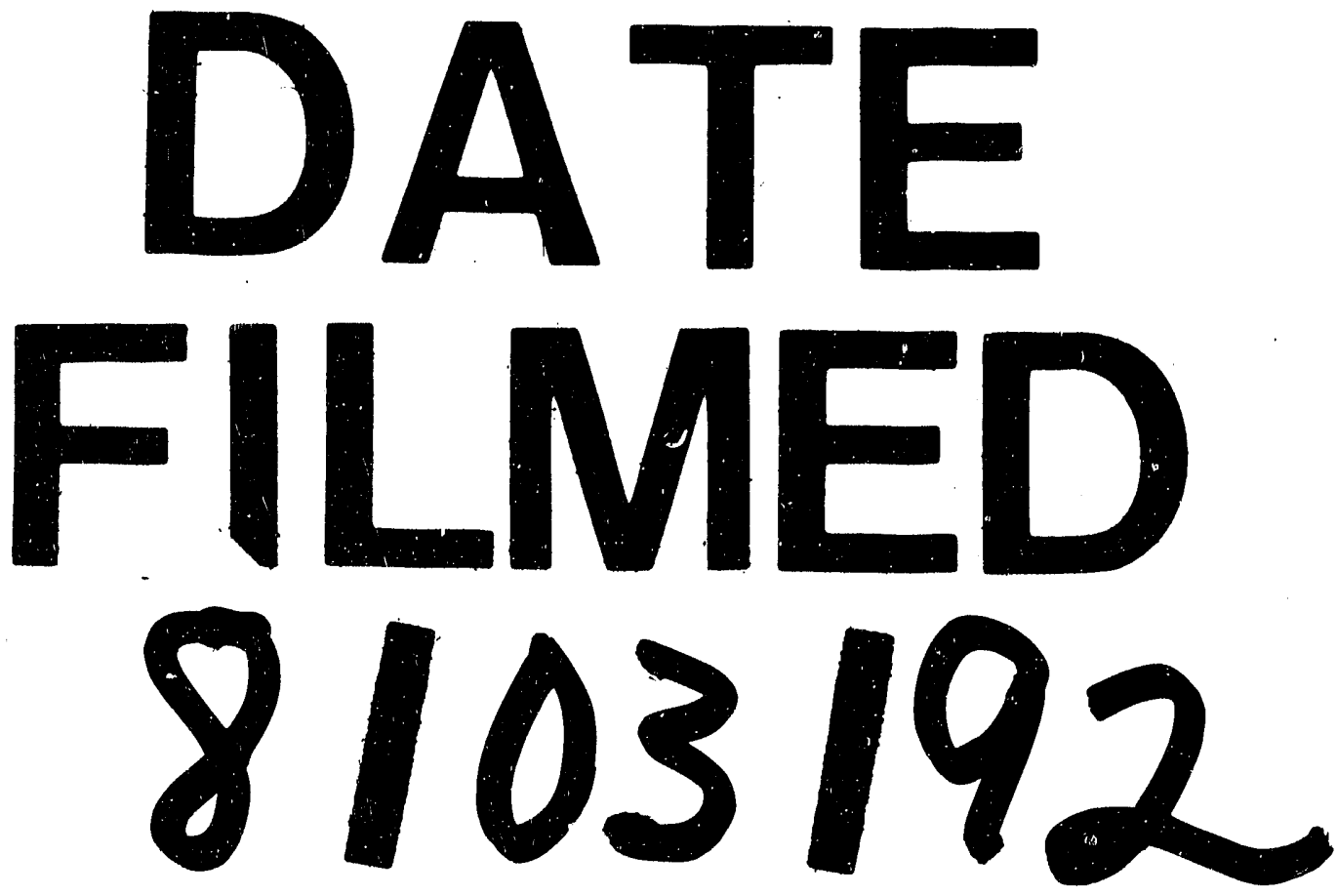
